Table S1: Results of weekly cycle analysis on $\mathrm{PM}_{2.5}$ and $\mathrm{PM}_{10-2.5}$ : black dots indicate a significant weekend effect magnitude (WEM) or weekly cycle (WCY) or 7-days periodicity (MTM) at a $95 \%$ confidence level. Results shown are from test application on the complete series, winter and summer by grouping data in 7-day weeks (this latter applies only to WEM and WCY).

\begin{tabular}{|c|c|c|c|c|c|c|c|}
\hline \multirow[t]{2}{*}{ Station } & \multicolumn{3}{|c|}{ Complete Series } & \multicolumn{2}{|c|}{ Winter } & \multicolumn{2}{|c|}{ Summer } \\
\hline & WEM & WCY & MTM & WEM & WCY & WEM & WCY \\
\hline \multicolumn{8}{|c|}{$\mathrm{PM}_{2.5}$} \\
\hline \multicolumn{8}{|l|}{ Alessandria } \\
\hline Ballirana & & - & & - & & & \\
\hline Bergamo & - & - & & & & - & - \\
\hline Besenzone & - & - & & & & $\bullet$ & $\bullet$ \\
\hline Biella & & $\bullet$ & & & & & \\
\hline \multicolumn{8}{|l|}{ Bologna G.M. } \\
\hline Bologna P.S.F. & & - & & & & - & - \\
\hline Borgofranco & & - & & & & • & \\
\hline Brescia & $\bullet$ & $\bullet$ & & & & $\bullet$ & $\bullet$ \\
\hline Calusco d'Adda & $\bullet$ & $\bullet$ & & & & $\bullet$ & $\bullet$ \\
\hline Casirate d'Adda & - & - & & & & • & - \\
\hline Castano Primo & & $\bullet$ & & & & & - \\
\hline Chivasso & - & - & & & & $\bullet$ & $\bullet$ \\
\hline \multicolumn{8}{|l|}{ Cornale } \\
\hline Faenza & & - & & & & & \\
\hline Ferrara & & - & & & & & \\
\hline Forli' & & - & & & & & • \\
\hline Gavello & & $\bullet$ & & & & & $\bullet$ \\
\hline Guastalla & & & - & & & & $\bullet$ \\
\hline Jolanda di Savoia & & - & & & & & \\
\hline Langhirano & & - & & & & & - \\
\hline Leiní & - & - & & & & & \\
\hline Lodi & - & - & & & & - & - \\
\hline Mantova & $\bullet$ & - & & & & $\bullet$ & $\bullet$ \\
\hline Merate & - & - & & & & - & - \\
\hline Milano & • & - & & & & - & - \\
\hline Modena & $\bullet$ & $\bullet$ & & & & & $\bullet$ \\
\hline Mortara & $\bullet$ & $\bullet$ & & & & & \\
\hline \multicolumn{8}{|l|}{ Novara } \\
\hline Padova & & - & & & & & \\
\hline Parma & & - & - & & & & \\
\hline \multicolumn{8}{|l|}{ Piacenza } \\
\hline Ponti sul Mincio & - & - & & & & & \\
\hline Reggio Emilia & - & - & & & & - & \\
\hline Rimini & - & - & $\bullet$ & & & & \\
\hline \multicolumn{8}{|l|}{ San Clemente } \\
\hline Saronno & $\bullet$ & - & & & & $\bullet$ & $\bullet$ \\
\hline Schivenoglia & $\bullet$ & - & & & & & \\
\hline Seriate & - & - & & & & - & - \\
\hline \multicolumn{8}{|l|}{ S. Pietro C. } \\
\hline Torino C. & $\bullet$ & & & & & & \\
\hline Torino L. & - & - & & & & & - \\
\hline Vercelli & & - & & & & & \\
\hline \multicolumn{8}{|l|}{ Vinchio } \\
\hline \multicolumn{8}{|c|}{$\mathrm{PM}_{10-2.5}$} \\
\hline Borgofranco & - & - & $\bullet$ & & & - & - \\
\hline Brescia & $\bullet$ & $\bullet$ & $\bullet$ & - & - & - & - \\
\hline Calusco d'Adda & $\bullet$ & $\bullet$ & & $\bullet$ & $\bullet$ & $\bullet$ & $\bullet$ \\
\hline Casirate d'Adda & - & - & - & • & & - & • \\
\hline Lodi & - & - & & & & - & - \\
\hline Mantova & - & - & & & - & & \\
\hline Merate & - & - & - & - & - & - & - \\
\hline Milano & $\bullet$ & $\bullet$ & $\bullet$ & $\bullet$ & $\bullet$ & $\bullet$ & $\bullet$ \\
\hline Parma & $\bullet$ & - & $\bullet$ & & $\bullet$ & $\bullet$ & \\
\hline Ponti sul Mincio & - & - & & - & - & - & - \\
\hline Reggio Emilia & - & - & & • & - & • & - \\
\hline Rimini & $\bullet$ & - & - & $\bullet$ & - & $\bullet$ & - \\
\hline Saronno & - & - & & $\bullet$ & - & • & - \\
\hline Schivenoglia & - & - & & & & & - \\
\hline Torino L. & $\bullet$ & $\bullet$ & & $\bullet$ & $\bullet$ & $\bullet$ & $\bullet$ \\
\hline
\end{tabular}




\section{Complete series}
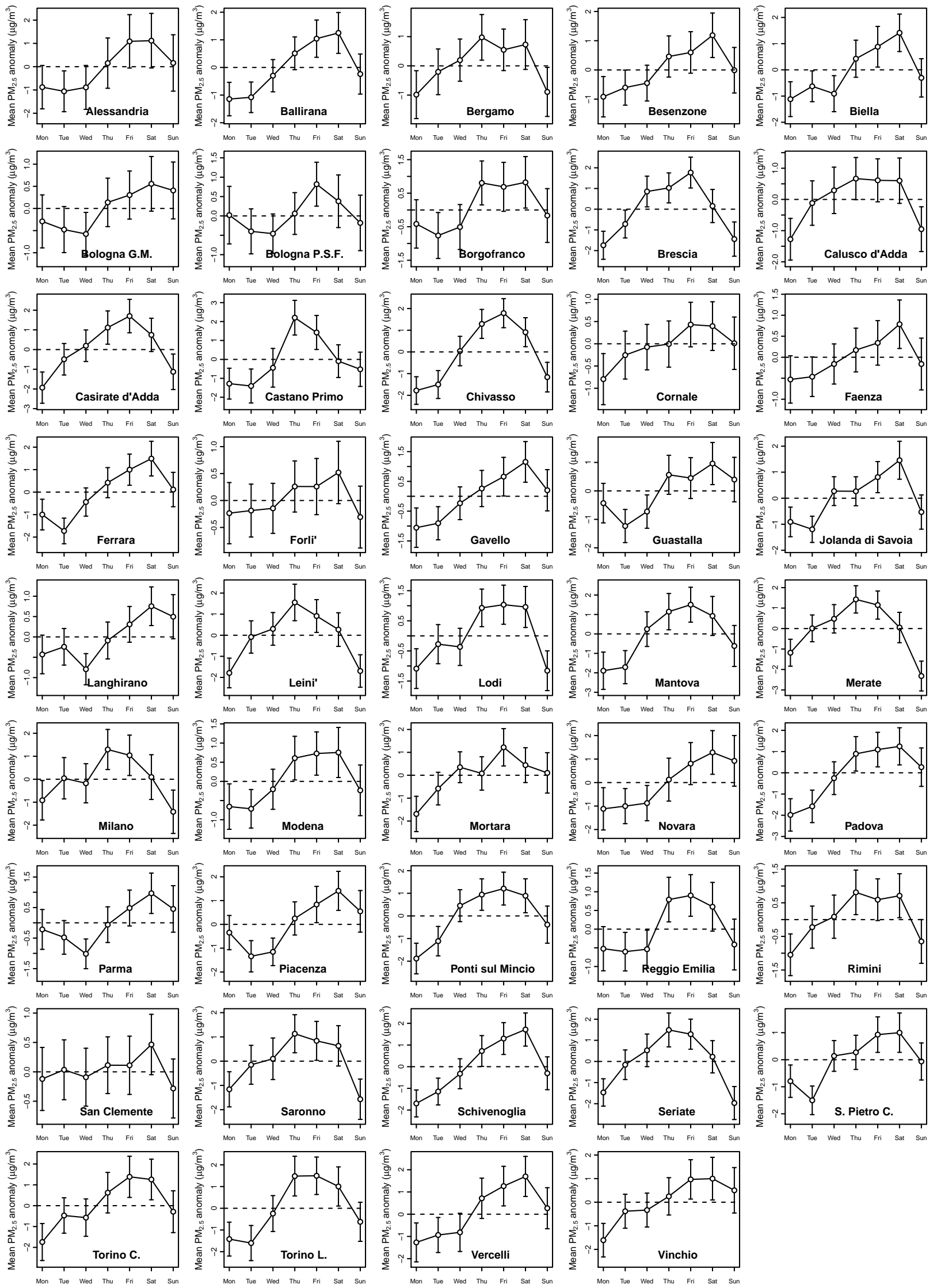

Figure S1: 7 day week mean $\mathrm{PM}_{2.5}$ anomaly for all sites listed in table 1 (vertical bars indicate standard deviation). 


\section{Complete series}
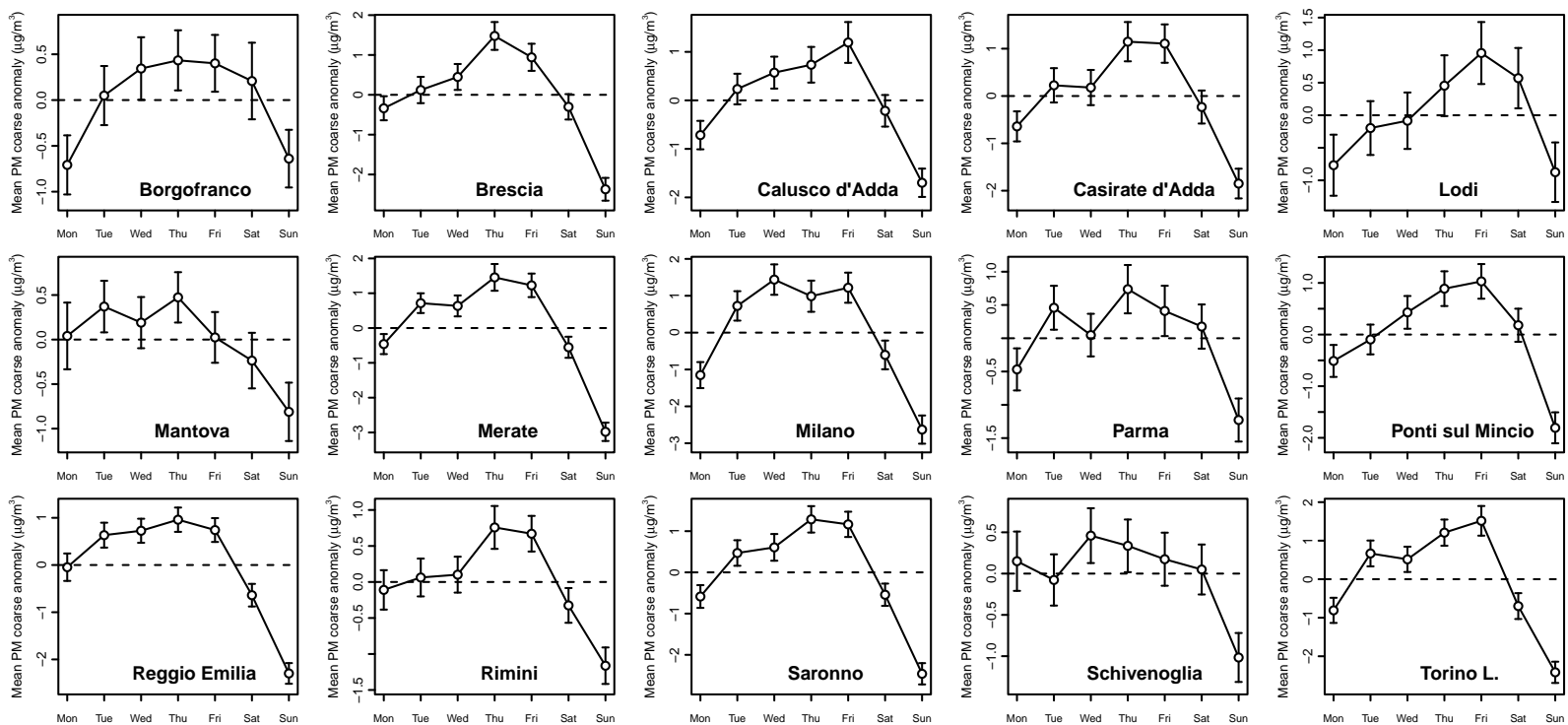

Figure S2: 7 day week mean $\mathrm{PM}_{10-2.5}$ anomaly for all sites listed in boldface in table 1 (vertical bars indicate standard deviation).

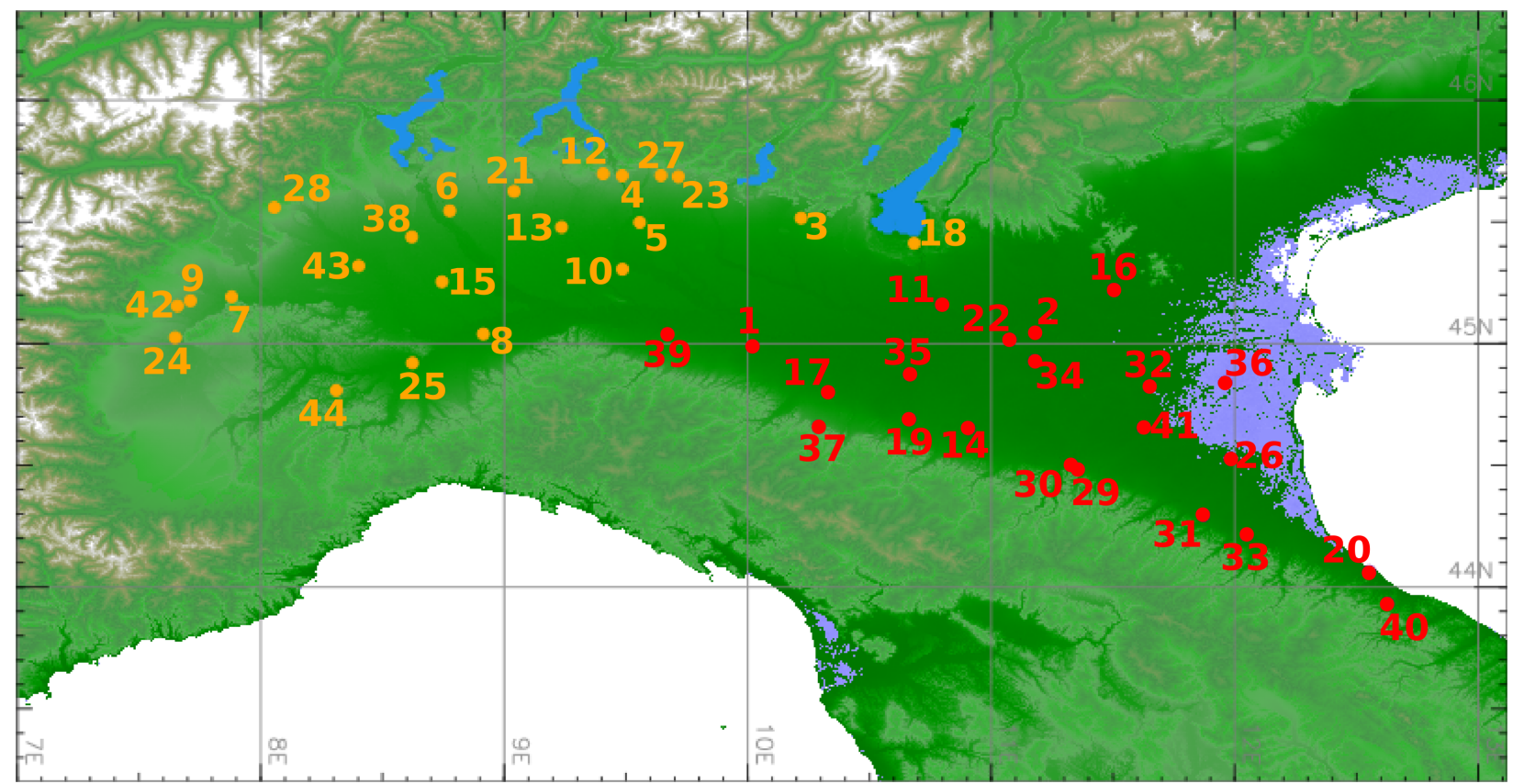

Figure S3: Map result for cluster analysis using partition around medoids algorithm: sites within the same cluster have the same colour. Results of cluster analysis using a divisive algorithm are in Figure 1. Key for ID number is found in Table 1. More details are in sections 2.3 and 3.3. 

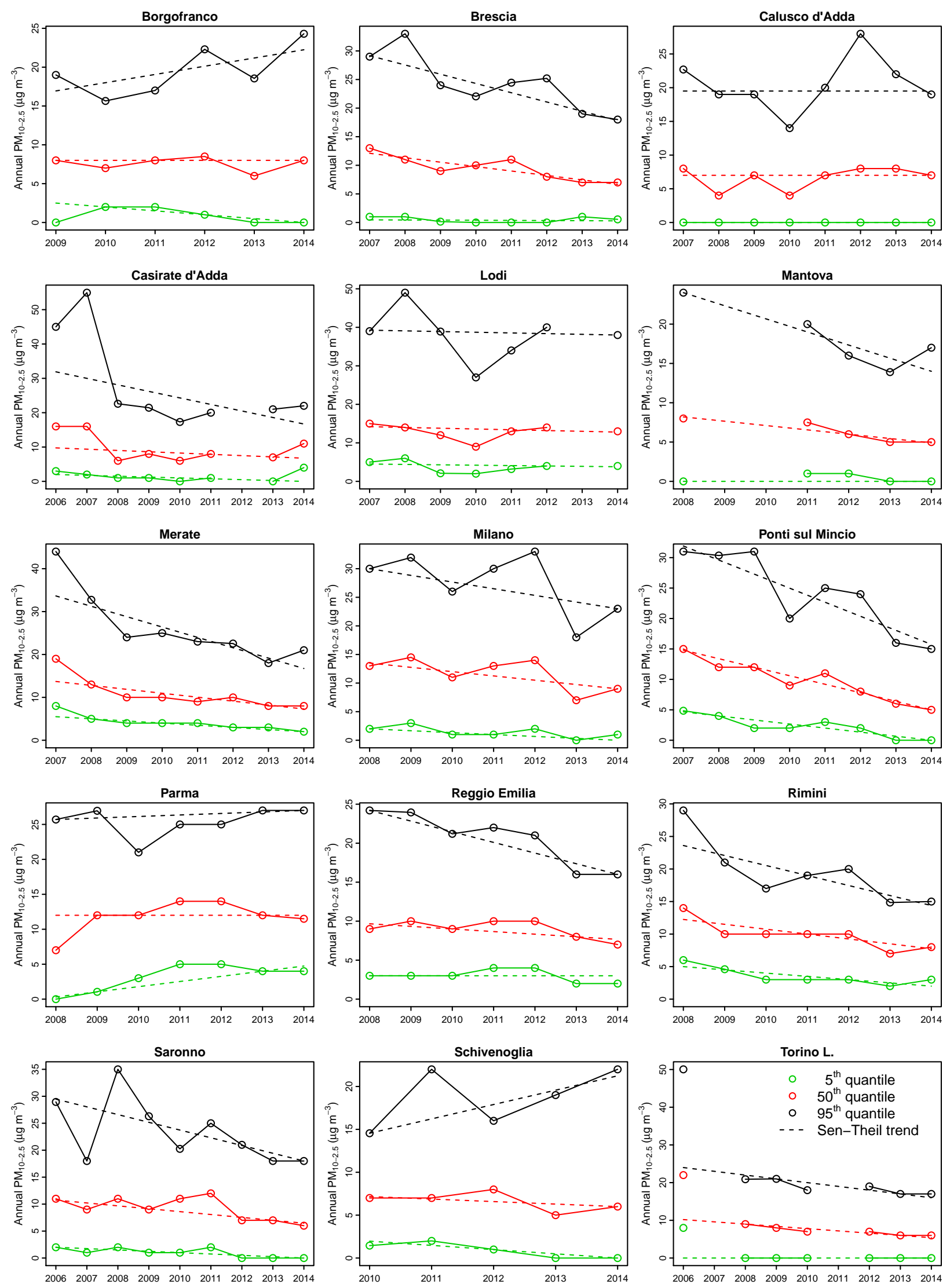

Figure S4: Annual quantile and Sen-Theil trend for daily $\mathrm{PM}_{10-2.5}$. Slope estimate and Significance are found in table 3 . 

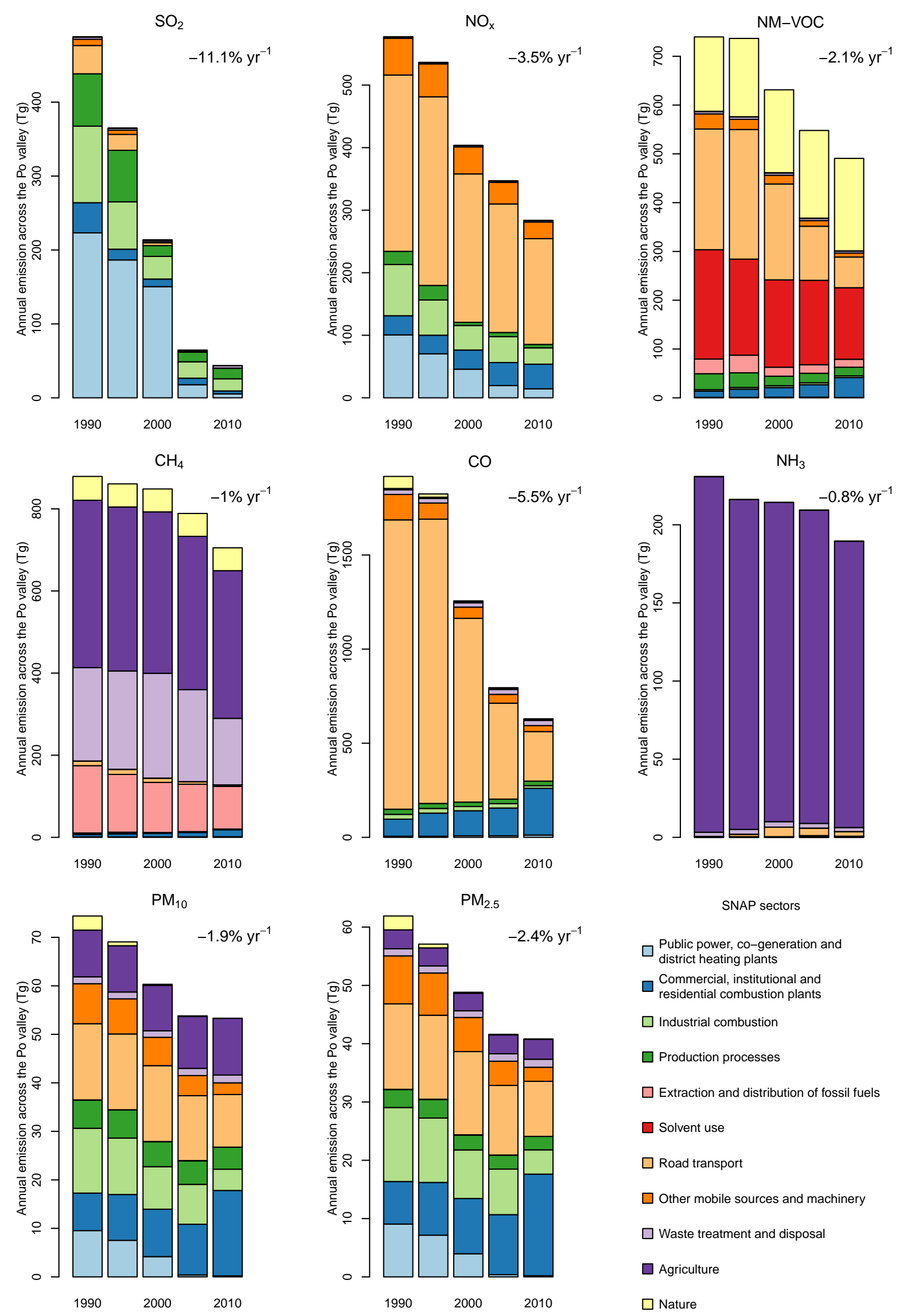

Figure S5: Emissions of selected compounds from all provinces on the Po valley, segregated by SNAP factor, over the period 1990-2010. 


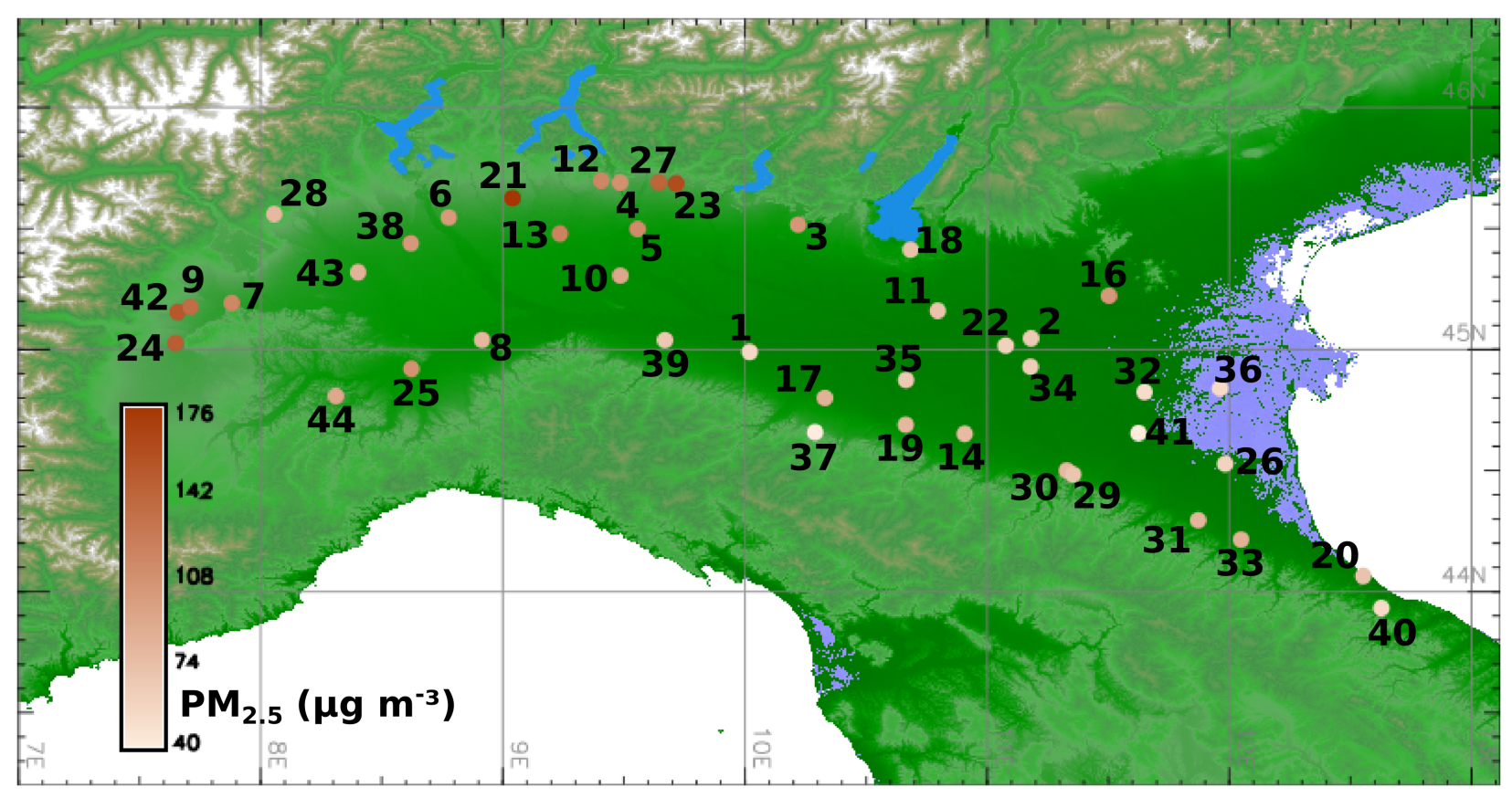

Figure S6: Maximum daily $\mathrm{PM}_{2.5}$ observed over the Po valley over the January pollution episode.

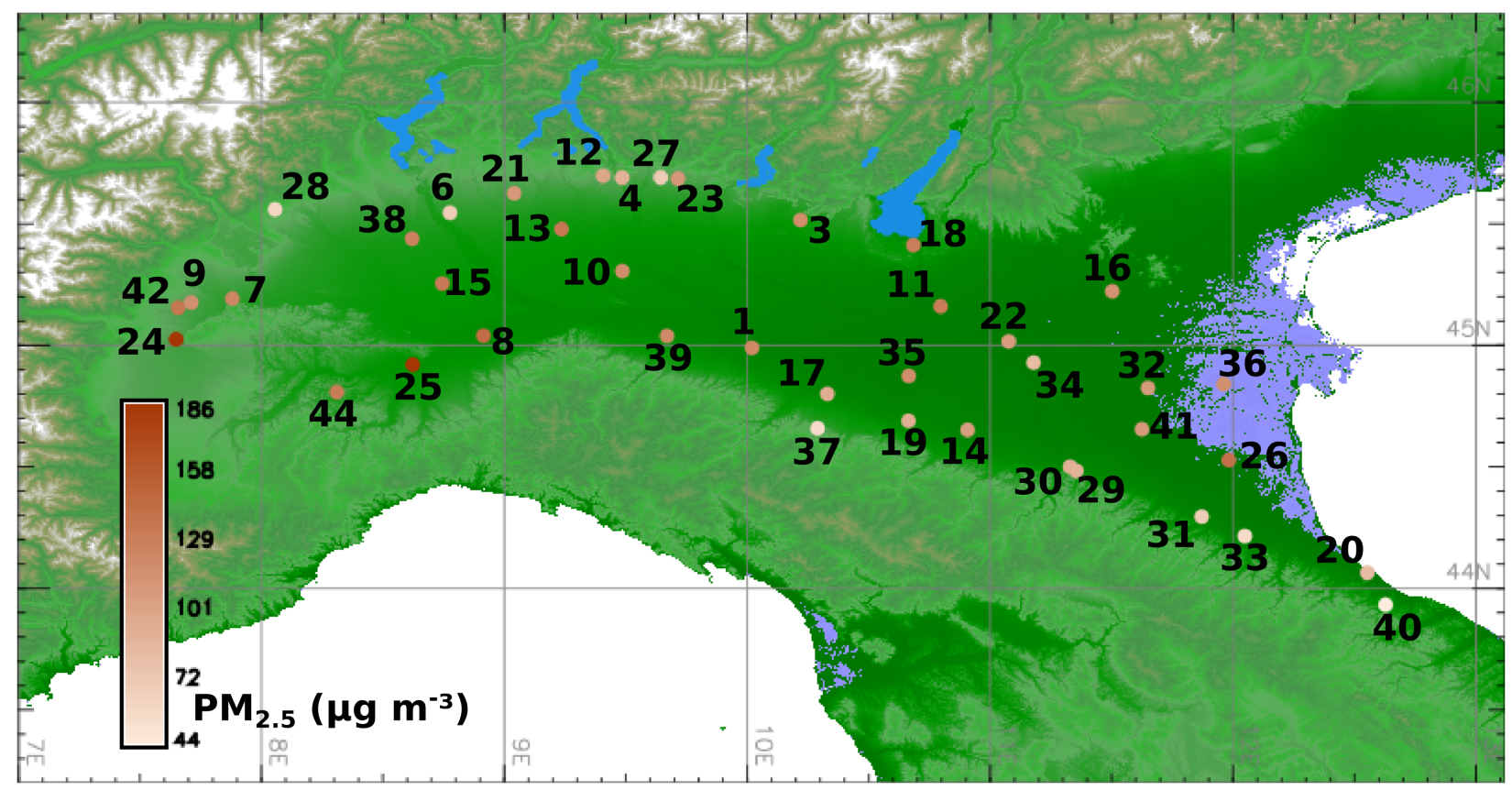

Figure S7: Maximum daily $\mathrm{PM}_{2.5}$ observed over the Po valley over the February pollution episode. 